\title{
Lúpus Induzido por Drogas - Da Imunologia Básica à Aplicada
}

\section{Drug-Induced Lupus - From Basic to Spplied Immunology}

\author{
Licia Maria Henrique da $\operatorname{Mota}^{(1)}$, Gustavo Pavlik Haddad(2), Rodrigo Aires Corrêa Lima ${ }^{(3)}$, \\ Jozélio Freire de Carvalho(4), Maria Imaculada Muniz-Junqueira ${ }^{(5)}$, \\ Leopoldo Luiz dos Santos Neto ${ }^{(6)}$, Francisco Aires Corrêa Lima ${ }^{(7)}$
}

\section{RESUMO}

O lúpus induzido por drogas (LID) é descrito como o desenvolvimento de sintomas semelhantes ao do lúpus eritematoso sistêmico idiopático, temporalmente relacionado à exposição a drogas, havendo, comumente, a resolução do quadro com a suspensão do medicamento desencadeante. A associação mais clássica é feita com a procainamida e a hidralazina. Recentemente, com a introdução de novas drogas na prática clínica, tem sido relatado um aumento no número de medicamentos implicados como causadores da doença, e a lista atual inclui quase uma centena de drogas relacionadas à ocorrência de LID. Embora descrito há mais de 60 anos, o mecanismo imunológico básico do LID ainda não está totalmente compreendido. Há várias hipóteses para o processo de indução de auto-imunidade pelas drogas, e o fenômeno geralmente é interpretado como uma inapropriada ativação do sistema imunitário. Entre as diversas teorias propostas, as mais aceitas são: a inibição da metilação do ácido desoxirribonucléico (DNA) por algumas drogas, o que permitiria a ativação das células T; a oxidação de certas substâncias pelos monócitos, gerando metabólitos ativos que ocasionariam ativação das células apresentadoras de antígenos e/ou a interferência dos metabólitos de determinadas drogas com a tolerância do sistema imune. Novos estudos são necessários para a melhor compreensão da imunopatogenia do LID, objetivando desenvolver tratamentos específicos com base no melhor conhecimento dos mecanismos patogênicos.

Palavras-chave: lúpus induzido por drogas, imunologia, lúpus eritematoso sistêmico, droga, auto-imunidade.

\begin{abstract}
Drug-induced lupus (DIL) has been described as the development of idiopathic systemic lupus erythematous-like symptoms, temporarily associated to the exposition to drugs, and as a rule, the condition is improved with the suspension of the triggering medication. The most classical association is with procainamide and hydralazine. Recently, with the introduction of new drugs in the clinical practice, an increase on the number of medications associated with the occurence of the disease has been reported, and the current list includes almost one hundred drugs associated to the occurrence of DIL. The basic DIL immunologic mechanism, although described for more than 60 years, is not yet fully understood. There are several hypotheses for the druginduced autoimmunity process, and the phenomenon is generally interpreted as an inappropriate activation of the immune system. Among the several theories proposed, the most accepted ones are: the inhibition of the DNA methylation by some drugs, what would allow the activation of T-cells; the oxidation of some substances by monocytes, what would generate active metabolites that in turn would lead to the activation of antigen-presenting cells and/or the interference of the metabolites of some drugs with the immune system tolerance. Further studies should be conducted in order to elucidate the DIL immunopathogeny with the objective of developing specific treatments based on the better knowledge on the pathogenic mechanisms.
\end{abstract}

Keywords: drug-induced lupus, immunology, systemic lupus erythematous, drug, autoimmunity.

\footnotetext{
Recebido em 27/03/07. Aprovado, após revisão, em 29/09/07. Declaramos a inexistência de conflitos de interesse.

Serviço de Reumatologia do Hospital Universitário de Brasília da Universidade de Brasília (1, 2, 3, 6, 7); Serviço de Reumatologia do Hospital das Clínicas da Faculdade de Medicina da Universidade de São Paulo (4); Laboratório de Imunologia Celular da Faculdade de Medicina da Universidade de Brasília (5).

1. Médica reumatologista, responsável pelos ambulatórios de Lúpus Eritematoso Sistêmico e Artrite Reumatóide Inicial do Serviço de Reumatologia do Hospital Universitário de Brasília - Universidade de Brasília (UnB)

2. Médico-residente em Reumatologia do Serviço de Reumatologia do Hospital Universitário de Brasília (UnB).

3. Médico reumatologista do Serviço de Reumatologia do Hospital Universitário de Brasília (UnB) e responsável pelo Ambulatório de Artrite Reumatóide Inicial do Serviço de Reumatologia do Hospital de Base do Distrito Federal (HBDF).

4. Médico, doutor em reumatologia, Responsável pelo Ambulatório de Síndrome Antifosfolípide do Serviço de Reumatologia do Hospital das Clínicas da Faculdade de Medicina da Universidade de São Paulo (HC-FMUSP).

5. Professora-associada de Imunologia e doutora em Imunologia e Genética Aplicadas pela Universidade de Brasília (UnB)

6. Professor adjunto de Clínica Médica e do Serviço de Reumatologia do Hospital Universitário de Brasília (HUB).

7. Médico reumatologista, responsável pelo Ambulatório de Colagenoses do Serviço de Reumatologia do Hospital Universitário de Brasília (UnB)

Endereço para correspondência: Licia Maria Henrique da Mota, SHIS OI 23, conj. 2, casa 9, Lago Sul, CEP 71660-020, Brasília, DF, e-mail: licia@unb.br
} 


\section{INTRODUÇÃO}

O lúpus induzido por drogas (LID) é definido como o lúpus eritematoso sistêmico (LES) idiopático relacionado à exposição contínua a fármacos (por mais de 30 dias), havendo, normalmente, resolução do quadro com a suspensão do medicamento desencadeante ${ }^{(1)}$.

O primeiro relato de LES induzido pelo uso de um medicamento, a sulfadiazina, foi feito em $1945^{(2)}$. A introdução de novas drogas na prática clínica tem sido acompanhada pelo aumento no número de medicamentos implicados como desencadeantes dessa condição patológica ${ }^{(3)}$.

Ainda não se conhecem os mecanismos envolvidos na fisiopatogenia do LID. Dados experimentais apontam para: a inibição da metilação do ácido desoxirribonucléico (DNA); a ativação de monócitos e distúrbios dos metabólitos de determinadas drogas no processo de tolerância do sistema imunitário ${ }^{(4)}$.

Em todas as situações propostas, uma modificação molecular específica desencadearia a ativação do sistema imunitário, resultando em auto-imunidade. O mecanismo parece ser multifatorial.

\section{CARACTERÍSTICAS EPIDEMIOLÓGICAS E CLÍNICAS DO LID}

Estima-se uma incidência de 15 mil a 20 mil casos de LID por ano em todo o mundo ${ }^{(5)}$. Considera-se que mais de $10 \%$ dos casos de LES são droga-induzidos, estimando-se em quase 100 o número de medicações envolvidas ${ }^{(6)}$.

O LID, diferentemente do LES, é mais comum na raça caucasiana, sendo raro nos negros; apresenta igual ocorrência entre os sexos, e a idade média de surgimento dos sintomas é maior do que a relatada no $\operatorname{LES}^{(7)}$.

O quadro clínico é de instalação insidiosa e pode ser semelhante ao do LES, lúpus eritematoso cutâneo subagudo e crônico ${ }^{(8)}$. O tempo decorrido entre a exposição à droga e a ocorrência dos sintomas varia de 30 dias a vários anos ${ }^{(9)}$. A confirmação definitiva dada é pela recidiva do quadro após reexposição à droga, o que não é necessário, por razões éticas. Sintomas semelhantes aos do lúpus idiopático, como artralgia, mialgia, febre e, ocasionalmente, pleurite e pericardite, podem ser observados ${ }^{(10)}$.

As lesões de pele e a artralgia são muito comuns tanto no LID quanto no LES, entretanto, diferentemente do LES, o acometimento do sistema nervoso central e renal é bastante incomum no LID. A presença de eritema nodoso, púrpuras ou pápulas eritematosas é mais comum na forma induzida por drogas do que no lúpus idiopático. Eritema malar, alopecia, lesões discóides, aftas orais, fotossensibilidade e fenômeno de Raynaud são pouco observados no LID ${ }^{(11)}$. O envolvimento sistêmico grave é raro, embora já tenha sido relatado. É possível a existência de lesões cutâneas características do lúpus eritematoso cutâneo subagudo, inclusive com a presença de anti-SS-A/Ro e anti-SS-B/La ${ }^{(12)}$.

$\mathrm{O}$ acometimento hematológico, expresso pela anemia, leucopenia ou ambos, ocorre em $25 \%$ a $33 \%$ dos casos. $\mathrm{O}$ teste de Coombs pode ser positivo em um terço dos pacientes, principalmente após o uso de procainamida, e é raro nas formas desencadeadas pela hidralazina. A plaquetopenia é um evento pouco freqüente ${ }^{(5)}$.

Assim como no LES, nos casos de LID também há freqüência elevada de produção de auto-anticorpos, principalmente os anticorpos antinúcleo (AAN), detectados na imunofluorescência ${ }^{(13)}$. O padrão homogêneo da distribuição dos anticorpos é o mais observado, em virtude da reatividade contra proteínas do grupo histona, sendo sua ocorrência maior entre mulheres ${ }^{(14)}$. Eventualmente, os AAN podem estar ausentes no LID $^{(15)}$.

Os anticorpos anti-histona, anti-DNA dupla-hélice (anti-dsDNA), antifator de necrose tumoral (TNF), antimieloperoxidase, antielastase e anticardiolipina induzidos pelas drogas apresentam negativação dos títulos após a retirada destas ${ }^{(16,17)}$.

Muitos casos de LID apresentam expressão clínica moderada e são autolimitados, embora outros possam ameaçar a vida do paciente e ter características clínicas indistinguíveis do LES idiopático.

A indução do LID tem relação com a dose e a duração do tratamento para cada droga, e para a procainamida a dose média de indução é de $1,5 \mathrm{~g}$, alcançada após o período de 12 a 24 meses de uso; e para a hidralazina, a dose é de $400 \mathrm{mg} /$ dia, e o tempo de exposição médio é de 17 a 26 meses. Entretanto, há diversos casos descritos com doses menores dos medicamentos e tempo de exposição variável ${ }^{(18)}$.

O tratamento baseia-se essencialmente no reconhecimento da condição clínica induzida pelo medicamento e imediata suspensão da droga. Nas formas mais graves ou nos casos de envolvimento pleuropericárdico significante, o uso de prednisona $0,5 \mathrm{mg}$ a $1 \mathrm{mg} / \mathrm{kg} /$ dia pode ser necessário ${ }^{(19)}$. Nas condições refratárias, o tratamento deve seguir as recomendações para o manejo do lúpus idiopático, inclusive podendo ser indicado o uso de drogas imunossupressoras ${ }^{(20)}$.

As principais características clínicas e laboratoriais que diferenciam o LID do LES estão listadas na tabela 1. 
São fatores de risco associados à ocorrência do LID: ser acetilador lento ${ }^{(21)}$, apresentar antígenos leucocitários humanos (HLA) DR4 ${ }^{(22)}$, HLA DR0301 ${ }^{(23)}$ e presença do alelo do complemento C3 null(24).

\section{TABELA 1}

COMPARAC̆̃̃ DOS PRINCIPAIS ACHADOS CLÍNICOS E LABORATORIAIS ENTRE O LID E O LES IDIOPÁTICO

\begin{tabular}{lcc}
\hline CARACTERÍSTICA & LID & LES idiopático \\
\hline Idade & Mais velhos & Idade reprodutiva \\
\hline Relação mulher:homem & $1: 1$ & $9: 1$ \\
\hline Gravidade & Leve & Variável \\
\hline Envolvimento SNC & Não & Sim \\
\hline Envolvimento renal & Não & Sim \\
\hline Pele & $\begin{array}{c}\text { Eritema nodoso, } \\
\text { púrpuras e lúpus } \\
\text { subagudo }\end{array}$ & $\begin{array}{c}\text { Eritema malar, } \\
\text { fotossensibilidade, } \\
\text { aftas orais }\end{array}$ \\
\hline Fenômeno de Raynaud & Raro & Comum \\
\hline Anticorpos anti-histona & $>95 \%$ & $50 \%$ \\
Anticorpos anti-DNA & $<5 \%$ & $50 \%$ a 70\% \\
\hline Prognóstico & $\begin{array}{c}\text { Remissão com a } \\
\text { suspensão da droga } \\
\text { indutora }\end{array}$ & Crônico, recorrente \\
\hline
\end{tabular}

Adaptado da referência 20.

\section{DROGAS IMPLICADAS NA OCORRÊNCIA DE LID}

A procainamida e a hidralazina são duas drogas classicamente associadas ao LID. Com a utilização durante 12 meses desses medicamentos, em doses convencionais, observa-se a ocorrência de LID em aproximadamente $20 \%$ dos pacientes em uso de procainamida e em $5 \%$ a $8 \%$ daqueles em uso de hidralazina. Com as demais drogas, a freqüência é menor, relatando-se a ocorrência de fenômenos de auto-imunidade em menos de $1 \%$ dos pacientes tratados $^{(6)}$.

As drogas associadas ao LID podem ser classificadas em 4 grupos, de acordo com o nível de evidência científica disponível da relação causal: grupo I, relação considerada "definitiva" (estudos controlados); grupo II, relação "provável" (estudos não controlados); grupo III, "possível" (relato ou séries de casos); grupo IV, dados de iniciais ${ }^{(6)}$. A tabela 2 resume a classificação das drogas relacionadas à ocorrência do LID.

No grupo IV, incluem-se os novos agentes biológicos, especialmente os bloqueadores do fator de necrose tumoral (TNF). Há registro da ocorrência de auto-anticorpos
TABELA 2

ClassificaÇÃo das DROGas RELACIONADAS À OCORRÊNCIA DE LID

\begin{tabular}{|c|c|}
\hline Grupo & Drogas \\
\hline $\begin{array}{l}\text { Grupo I: Drogas } \\
\text { definitivamente } \\
\text { capazes de } \\
\text { induzir lúpus }\end{array}$ & $\begin{array}{l}\text { Hidralazina } \\
\text { Procainamida } \\
\text { Isoniazida } \\
\text { Metildopa } \\
\text { Clorpromazina } \\
\text { Quinidina } \\
\text { Minociclina }\end{array}$ \\
\hline $\begin{array}{l}\text { Grupo II: Drogas } \\
\text { provavelmente } \\
\text { capazes de } \\
\text { induzir lúpus }\end{array}$ & $\begin{array}{l}\text { Sulfassalazina } \\
\text { Anticonvulsivantes (carbamazepina, etosuximida, } \\
\text { fenitoína, difenilhidantoína, primidona, trimetadiona, } \\
\text { valproato) } \\
\text { Drogas antitireoidianas (propiltiouracil, metimazol) } \\
\text { Terbinafina } \\
\text { Estatinas (sinvastatina, lovastatina, fluvastatina) } \\
\text { Penicilamina } \\
\text { Betabloqueadores (propranolol, atenolol, acebutolol, } \\
\text { labetalol, pindolol, metoprolol, timolol) } \\
\text { Hidroclorotiazida } \\
\text { Interferon- } \alpha \text { (IFN- } \alpha \text { ) } \\
\text { Fluorouracil }\end{array}$ \\
\hline $\begin{array}{l}\text { Grupo III: } \\
\text { Drogas possivel- } \\
\text { mente capazes } \\
\text { de induzir lúpus }\end{array}$ & $\begin{array}{l}\text { Sais de ouro } \\
\text { Antibióticos (penicilina, estreptomicina, tetraciclina, } \\
\text { ciprofloxacina, rifampicina) } \\
\text { Griseofulvina } \\
\text { Fenilbutazona } \\
\text { Estrógenos (anticonceptivos orais e terapia de reposi- } \\
\text { ção hormonal) } \\
\text { Reserpina } \\
\text { Lítio } \\
\text { Ácido paraaminosalicílico } \\
\text { Captopril } \\
\text { Bloqueadores dos canais de cálcio } \\
\text { Clonidina } \\
\text { Hidroxiuréia } \\
\text { Genfibrozila }\end{array}$ \\
\hline $\begin{array}{l}\text { Grupo IV: Drogas } \\
\text { recentemente } \\
\text { relatadas como } \\
\text { capazes de } \\
\text { induzir lúpus }\end{array}$ & $\begin{array}{l}\text { Interleucina-2 } \\
\text { Clobazam } \\
\text { Clozapine } \\
\text { Tocainida } \\
\text { Lisinopril } \\
\text { Anti-TNF (etanercepte, infliximabe, adalimumabe) } \\
\text { Efalizumabe } \\
\text { Zafirlucaste } \\
\text { Bupropiona }\end{array}$ \\
\hline
\end{tabular}

Adaptado da referência 6.

e/ou LID clínico com infliximabe ${ }^{(25,26)}$, etanercepte ${ }^{(27)}$, adalimumabe $^{(28,29)}$ e efalizumabe ${ }^{(30)}$, independentemente da doença de base. Entre eles são descritos anticorpos antinúcleo, anti-ribonucleoproteína (anti-RNP), anti-dsDNA, anticardiolipina e anticorpos contra o antígeno Smith $(\text { anti-Sm })^{(31)}$. A indução com terapia anti-TNF em curto prazo se correlaciona à produção desses auto-anticorpos. Não há associação a outros sinais ou alterações sorológicas sugestivas de LES $^{(32)}$. Embora alguns trabalhos sugiram que a ocorrência de auto-anticorpos seja maior com o uso do infliximabe do que com o uso do etanercepte ${ }^{(33)}$, os dados ainda são controversos ${ }^{(26,34)}$. 


\section{IMUNOPATOGENIA DO LID}

A imunopatogenia do LID ainda é pouco conhecida, no entanto, apresenta uma série de peculiaridades em relação a outras condições droga-induzidas, sendo possível que os aspectos imunopatogênicos relacionados a essa doença sejam derivados de outras situações clínicas ${ }^{(4)}$.

Os principais mecanismos envolvidos são:

\section{1) METABOLISMO OXIDATIVO DAS DROGAS}

Diversas drogas implicadas na ocorrência do LID, pertencentes às mais diversas classes farmacológicas, sofrem metabolismo oxidativo por meio de neutrófilos ativados. Nesse grupo, podemos citar a procainamida, a hidralazina e outras. A taxa de acetilação (acetiladores lentos versus acetiladores rápidos) não é um fator de risco para o LES idiopático. No entanto, tem sido considerado que a acetilação lenta é um dos fatores que predispõe à ocorrência de LID em pacientes em uso de hidralazina e procainamida ${ }^{(35)}$.

A ativação dos neutrófilos por partículas opsonizadas ou certas substâncias solúveis estimula a produção de radicais livres. Da reação do $\mathrm{H}_{2} \mathrm{O}_{2}$ com a procainamida catalisada pela mieloperoxidase (MPO) liberada pelos neutrófilos, há a formação de procainamida - hidroxilamina (PAHA). Esse composto é capaz de ativar células mononucleares e tem sido associado aos mecanismos da doença auto-imune ${ }^{(36)}$.

\section{2) INTERFERÊNCIA DOS METABÓLITOS DAS DROGAS NA TOLERÂNCIA IMUNOLÓGICA E O PAPEL DA SELEÇÃO TÍMICA NO LID}

A interrupção da tolerância imunológica tem implicação na indução de auto-imunidade ${ }^{(37)}$. Tem-se demonstrado que a injeção de metabólitos ativos da procainamida diretamente no timo de ratos modifica a tolerância imunológica e induz a formação de diversos auto-anticorpos, processo muito semelhante ao observado em pacientes com lúpus induzido por procainamida ${ }^{(38)}$. Esses animais, no entanto, não desenvolveram evidência clínica de doença, o que leva a crer que outros fatores, além da modificação da tolerância, estejam envolvidos no desencadeamento do LID $^{(39)}$.

Um dos mecanismos propostos para a indução de autoimunidade pelo PAHA relaciona-se à seleção das células $\mathrm{T}$ $\mathrm{CD}^{+} \mathrm{CD}^{+}$no córtex do timo, expressando o receptor de célula T (TCR) e diferindo em afinidade para antígenos próprios $^{(36)}$.

Após diferenciação em células $\mathrm{T} \mathrm{CD}^{+}$maduras e migração para a periferia, as células $\mathrm{T}$ não tolerantes por influência da PAHA poderão ser ativadas. Ao encontrar uma célula $B$ cromatina específica, que se liga ao antígeno da célula T no seu MHC, a imunidade T-B é induzida, desencadeando auto-imunidade ${ }^{(36)}$.

Estudo realizado em pacientes entre a sexta e a oitava décadas de vida, que desenvolveram LID durante tratamento com procainamida, demonstrou a presença de células recentemente emigradas do timo, marcadas com $T$ cell receptor excision circles (TRECs), em sua população de linfócitos circulantes. Os níveis dessas células correlacionaram-se significantemente com a atividade do anticorpo anticromatina $^{(40)}$.

\section{3) INIBIÇÃO DA METILAÇÃO DO DNA}

A metilação do DNA é um determinante fundamental da estrutura da cromatina, exercendo potente efeito supressivo na expressão gênica ${ }^{(41)}$. Em geral, genes inativos são silenciados pela metilação, que tem como função a supressão de genes não-essenciais ou potencialmente deletérios às funções celulares, como é o caso da hipometilação/desmetilação do gene da perforina, induzindo a produção de perforina e do antígeno $\mathrm{l}$ associado à função leucocitária (LFAl). Essas duas substâncias estão aumentadas nos pacientes com lúpus idiopático ativo e nos pacientes com $\operatorname{LID}^{(42)}$.

A metilação tem sido implicada como um dos principais mecanismos envolvidos no desenvolvimento das células do sistema imunitário, controlando a recombinação da região do segmento gênico da junção de diversidade variável (VDJ) ${ }^{(43)}$, a expressão dos antígenos da superfície celular e a regulação da produção de citocinas durante a resposta imune $e^{(44)}$.

Inibição exógena da metilação do DNA da célula T tem sido implicada no desenvolvimento do $\operatorname{LID}^{(45)}$. O uso de inibidores da metilação do DNA, incluindo a procainamida e a hidralazina, causa uma doença semelhante ao lúpus em modelos animais ${ }^{(46)}$.

\section{4) EXPOSIÇÃO A ANTÍGENOS OCULTOS}

A exposição a antígenos próprios ocultos pode ser outro mecanismo pelo qual haveria a perda da tolerância periférica. Tem sido proposto que drogas como infliximabe poderiam ocasionar a liberação de partículas intracelulares que serviriam como imunógenos e originariam uma resposta imune inadequada ${ }^{(3)}$.

\section{5) INDUÇÃO DE APOPTOSE EM LINFOBLASTOS HUMANOS ATIVADOS}

Demonstrou-se que a estimulação de células mononucleares do sangue periférico com material apoptótico 
autólogo pode levar à formação de clones de células $\mathrm{T}$ histona-específicos in vitro. Essas células $\mathrm{T}$ poderiam estimular linfócitos $\mathrm{B}$ autólogos a produzirem anticorpos anti-dsDNA, ocasionando mais marcado influxo de material apoptótico, facilitando a indução de auto-imunidade e a produção de auto-anticorpos ${ }^{(47)}$.

Esse mecanismo foi especificamente proposto para o lúpus induzido por clorpromazina, embora também possa participar da imunopatogênese do desencadeamento de auto-imunidade por outras drogas ${ }^{(48)}$.

\section{PERSPECTIVAS DE UTILIZAÇÃO DOS CONHECIMENTOS IMUNOPATOGÊNICOS NO TRATAMENTO DO LID}

Os pontos mais desafiadores na imunopatogenia do LID são:

1) As drogas implicadas na etiologia do LID apresentam estruturas químicas e ações farmacológicas das mais diversas, apesar de as características laboratoriais e clínicas do LID serem essencialmente semelhantes, independentemente da droga indutora.

2) Exceto por sua ação farmacológica, as drogas são inertes em doses habituais. O LID é uma reação idiossincrática das drogas, não sendo previsto por nenhuma propriedade conhecida das drogas.

3) Em geral, são necessários meses a anos de exposição às drogas para o desencadeamento das manifestações.

Para que haja a formação dos compostos ativos, é necessária a desgranulação dos neutrófilos, liberando mieloperoxidase, assim como a produção do ânion superóxido $\left(\mathrm{O}_{2}{ }^{-}\right)$. Posteriormente, há a transferência de um átomo de oxigênio, resultando um novo composto reativo. A administração de uma droga que inibisse a desgranulação dos neutrófilos poderia auxiliar na investigação diagnóstica, na medida em que inibiria a formação dos compostos ativos.

Uma droga inibidora do processo de desgranulação e da formação do ânion superóxido $\mathrm{O}_{2}$ é a adenosina ${ }^{(49)}$. Portanto, a adenosina poderia ser utilizada para confirmar a hipótese do metabolismo oxidativo como mecanismo etiopatogênico comum, na medida em que inibiria a formação de compostos reativos indutores de auto-imunidade.

Os conhecimentos sobre a imunopatologia do LES levam ao questionamento sobre a segurança do uso de adjuvantes oleosos na fabricação de vacinas humanas e veterinárias, uma vez que alguns estudos apontam para uma possível indução de auto-imunidade pela adjuvância dos hidrocarbonetos ${ }^{(50)}$.

Outra proposta de aplicação dos conhecimentos da imunologia no tratamento do LID é o uso de peptídeos com base nas regiões determinantes de complementariedade (CDR) de anticorpos monoclonais anti-DNA murinos $\mathrm{e}$ humanos, que se ligam a um idiotipo comum (16/6 Id). O uso desses peptídeos melhora as manifestações de doença em camundongos com LES espontâneo ou induzido por droga, e sua utilização em seres humanos é ainda uma perspectiva $^{(51)}$.

\section{CONCLUSÃO}

O LID é uma condição descrita há mais de 60 anos, listando-se atualmente quase uma centena de drogas possíveis de serem desencadeantes. As características clínicas e laboratoriais dessa doença são similares ao LES idiopático, exceto pela melhora completa do quadro clínico com a suspensão da medicação. A maioria dos relatos publicados evidencia uma doença autolimitada, de leve a moderada gravidade. Mas já foi bem demonstrado que o quadro clínico pode ser grave, indistinguível do LES idiopático, com manifestações eventualmente fatais.

A introdução recente de novas drogas na prática clínica, incluindo os agentes biológicos, tem sido acompanhada por um aumento no número de casos de LID. A maioria das publicações sobre o uso de agentes biológicos e o surgimento de LID é composta por relato de casos, havendo necessidade de estudos randomizados controlados para melhor esclarecimento dessa condição.

Embora a patogênese do LID não seja completamente entendida, a predisposição genética tem importante papel na doença desencadeada por algumas drogas, particularmente aquelas que são metabolizadas pela acetilação. O LID mais provavelmente ocorre em pacientes acetiladores lentos, nos quais há uma redução geneticamente mediada da síntese de $\mathrm{N}$-acetiltransferase.

Há um grande número de mecanismos propostos para a indução de auto-imunidade droga-mediada, e o processo, de forma geral, é considerado uma ativação inapropriada do sistema imunitário.

Entre as hipóteses para a imunopatogênese do LID estão: a inibição da acetilação do DNA, ocasionando a ativação das células $\mathrm{T}$; a ação de metabólitos ativos que poderiam interferir com a tolerância, a seleção tímica e/ ou a ativação das células apresentadoras de antígenos, permitindo uma ativação generalizada e inapropriada do 
sistema imunitário; e a exposição a antígenos ocultos, que poderiam desencadear a resposta imune.

No entanto, nenhuma das teorias atualmente propostas explica, isoladamente, todo o complexo fenômeno imunopatológico do LID. Algumas das hipóteses restringem-se a processos desencadeados por uma droga ou um grupo farmacológico específico e são insuficientes para abranger as diversas medicações possivelmente implicadas na etiopatogênese da condição.

O mais provável é que múltiplos mecanismos estejam envolvidos, simultânea ou consecutivamente, e que drogas

\section{REFERÊNCIAS}

1. Adhami E: A predictive equation for drug-induced lupus. Med Hypotheses 61(4): 473-6, 2003.

2. Hoffman BJ: Sensitivity to sufadizine resembling acute disseminated lupus erythematosus. Arch Dermatol Physiol 51: 90-2, 1945.

3. Haraoui B, Keystone E: Musculoskeletal manifestations and autoimmune diseases related to new biologic agents. Curr Opin Rheumatol 18(1): 96-100, 2006.

4. Uetrecth J: Current trends in drug-induced autoimmunity. Autoimmun Rev 4: 309-14, 2005.

5. Atzeni F, Marrazza MG, Sarzi-Puttini P, Carrabba M: Druginduced lupus erythematosus. Reumatismo 55: 147-54, 2003.

6. Sarzi-Puttini P, Atzeni F, Capsoni F, Lubrano E, Doria A: Drug-induced lupus erythematosus. Autoimmunity 38(7): 507-18, 2005.

7. Antonov D, Kazandjieva J, Etugov D, Gospodinov D, Tsankov N: Drug-induced lupus erythematosus. Clin Dermatol 22: 157-66, 2004 .

8. Callen JP: Drug-induced cutaneous lupus erythematosus, a distinct syndrome that is frequently under-recognised. J Am Acad Dermatol 45: 315-6, 2001.

9. Pelizza L, De Luca P, La Pesa M, Minervino A: Drug-induced systemic lupus erythematosus after 7 years of treatment with carbamazepine. Acta Biomed 77(1): 17-9, 2006.

10. Weinstein A: Drug-induced systemic lupus erythematosus. Prog Clin Immunol 4: 1-21, 1980.

11. Grossman L, Barland P: Histone reactivity of drug-induced antinuclear antibodies. A comparison of symptomatic and asymptomatic patients. Arthritis Rheum 24: 927-31, 1981.

12. Srivastava M, Rencic A, Diglio G, et al.: Drug-induced, Ro/ SSA positive cutaneous lupus erythematosus. Arch Dermatol 139: 45-9, 2003.

13. Rubin RL, Teodorescu M, Beutner EH, Plunkett RW: Complement fixing properties of antinuclear antibodies distinguish drug-induced lupus from systemic lupus erythematosus. Lupus 13: 249-56, 2004.

14. Rubin RL, Waga S: Antihistone antibodies in systemic lupus erythematosus. J Rheumatol 13S: 118-26, 1987. distintas desencadeiem a auto-imunidade por processos distintos.

O reconhecimento do fato de a doença ser drogainduzida evita a realização de investigações desnecessárias e permite o manejo adequado do paciente, ou seja, descontinuação do agente desencadeante, adequada monitoração e/ou intervenção com ações preventivas apropriadas ${ }^{(52)}$.

As lacunas no conhecimento da imunologia básica do processo limitam sua aplicação na terapêutica da doença, havendo, atualmente, poucas propostas do uso dos conhecimentos imunológicos para o tratamento específico do LID.

15. Carter JD, Valeriano-Marcet J, Kanik KS, Vasey FB: Antinuclear antibody-negative, drug-induced lupus caused by lisinopril. South Med J 94: 1122-3, 2001.

16. Mohan AK, Edwards ET, Cote TR, et al.: Drug-induced systemic lupus erythematosus and TNF-alpha blockers. Lancet 360: 646, 2002.

17. Torffvit $\mathrm{O}$, Thysell H, Nassberger L: Occurrence of autoantibodies direct against myeloperoxidase and elastasein patients treated with hydralazine and presenting with glomerulonephritis. Hum Exp Toxicol 13: 563-7, 1994.

18. Hosftra A: Metabolism of hydralazine: Relevance to drug induced lupus. Drug Metab Rev 26: 135-8, 1994.

19. diFazano CS, Bertin P: The pharmacological management of drug-induced rheumatic disorders. Expert Opin Pharmacoter 10: 1623-31, 2001.

20. Vasoo S: Drug-induced lupus: an update. Lupus 15 (11): 757-61, 2006.

21. Mansilla-Tinoco R, Harland SJ, Ryan PJ, et al.: Hydralazine, antinuclear antibodies, and the lupus syndrome. Br Med J 289: 410-2, 1984.

22. Batchelor JR, Welsh KI, Tinoco RM, et al.: Hydralazine-induced systemic lupus erythematosus: influence of HLA-DR and sex on susceptibility. Lancet 1: 1107-9, 1980.

23. Gunnarson I, Nordmark B, Hassan BA, et al.: Development of lupusrelatedside effects in patients with early RA during sulphasalazine treatment - the role of IL-10 and HLA. Rheumatology 39: 886-93, 2000.

24. Speirs C, Chapel H, Fielder AL, Davel NJ, Batchelor JR: Complement system protein $\mathrm{C} 4$ and susceptibility to hydralazineinduced systemic lupus erythematosus. Lancet 1: 922-4, 1989.

25. Louis M, Rauch J, Armstrong M, Fitzcharles MA: Induction of autoantibodies during prolonged treatment with infliximab. J Rheumatol 30: 2557-62, 2003.

26. Benucci M, Li Gobbi F, Fossi F, Manfredi M, Del Rosso A: Druginduced lupus after treatment with infliximab in rheumatoid arthritis. J Clin Rheumatol 11(1): 47-9, 2005.

27. Shakoor N, Michalska M, Harris CA, Block JA: Drug-induced systemic lupus erythematosus associated with etanercept therapy. Lancet 359: 579-80, 2002.

28. Van Rijthoven AW, Bijlsma JW, Canninga-van Dijk M, Derksen $\mathrm{RH}$, van Roon JA: Onset of systemic lupus erythematosus 
after conversion of infliximab to adalimumab treatment in rheumatoid arthritis with a pre-existing anti-dsDNA antibody level. Rheumatology (Oxford) 45 (10): 1317-9, 2006.

29. Scheinfeld N: Adalimumab - a review of side effects. Expert Opin Drug Saf 4 (4): 637-41, 2005.

30. Bentley DD, Graves JE, Smith DI, Heffernan MP: Efalizumabinduced subacute cutaneous lupus erythematosus. J Am Acad Dermatol 54 (5): 242-3, 2006.

31. De Bandt M, Vittecoq O, Descamps V, Le Loet X, Meyer O: Anti-TNF alpha-induced systemic lupus erythematosus. Clin Rheumatol 22: 56-61, 2003.

32. De Rycke L, Baeten D, Kruithof E, Van den Bosch F, Veys EM, De Keyser F: The effect of TNF alpha blockade on the antinuclear antibody profile in patients with chronic arthritis: biological and clinical implications. Lupus 14(12): 931-7, 2005.

33. De Rycke L, Baeten D, Kruithof E, Van den Bosch F, Veys EM, De Keyser F: Infliximab, but not etanercept, induces IgM anti-double-stranded DNA autoantibodies as main antinuclear reactivity: biologic and clinical implications in autoimmune arthritis. Arthritis Rheum 52(7): 2192-201, 2005.

34. De Bandt M, Sibilia J, Le Loet X, et al.: Systemic lupus erythematosus induced by anti-tumour necrosis factor alpha therapy: a Frech national survey. Arthritis Res Ther 7(3): R545-51, 2005.

35. Jiang X, Khursigara G, Rubin RL: Transformation of lupus inducing drugs to cytotoxic products by activated neutrophils. Science 266: 810-3, 1994.

36. Grant DM, Morike K, Eichelbaum M, Meyer UA.: Acetylation pharmacokinetics. The slow acetylator phenotype is caused by decreased or absent arylamine $\mathrm{N}$-acetyltransferase in human liver. J Clin Invest 85: 968-72, 1990.

37. Rubin RL: Drug-induced lupus. Toxicology 209(2): 135-47, 2005.

38. Rubin RL, Kretz-Rommel A: Persistence of autoreactive T cells drive is required to elicit anti-chromatin antibodies in a murine model of drug-induced lupus. J Immunol 162: 813-20, 1999.

39. Kretz-Rommel A, Duncan SR, Rubin RL: Autoimmunity caused by disruption of central $\mathrm{T}$ cell tolerance: a murine model of drug-induced lupus. J Clin Invest 99: 1888-96, 1997.
40. Kretz-Rommel A, Rubin RL: Disruption of positive selection of thymocytes causing autoimmunity. Nat Med 6: 298-305, 2000.

41. Ballestar E, Esteller M, Richardson BC: The epigenetic face of systemic lupus erythematosus. J. Immunol 176(12): 7143-7, 2006.

42. Kaplan MJ, Lu Q, Wu A, Attwood J, Richardson B: Demethylation of promoter regulatory elements contributes to perforin overexpression in $\mathrm{CD}^{+}$lupus T cells. J Immonol 172: 3652-61, 2004.

43. Abbas AK, Lichtman AH, Pober JS: Cellular and Molecular Immunology, 4 th Edition., Philadelphia, Editor W. B. Saunders Company, Chap. 7: 125-60, 2000.

44. Yung R, Richardson BC: Role of T cell DNA methylation in lupus sundromes. Lupus 3(6): 487-91, 1994.

45. Bonilla-Henao V, Martinez R, Sobrino F, Pintado E: Different signaling pathways inhibit DNA methylation activity and upregulate IFN-\{gamma\} in human lymphocytes. J Leukoc Biol 78(6): 1339-46, 2005.

46. Richardson B, Ray D, Yung R: Murine models of lupus induced by hypomethylated T cells. Methods Mol Med 102: 285-94, 2004.

47. Herrmann M, Voll RE, Zoller OM, Hagenhofer M, Ponner BB, Kalden JR: Impaired phagocytosis of apoptotic cell material by monocyte-derived macrophages from patients with systemic lupus erythematosus. Arthritis Rheum 41: 1241-50, 1998.

48. Hieronymus T, Grötsch P, Blank N, et al.: Chlorpromazine induces apoptosis in activated human lymphoblasts, Arthritis Rheum 43(9): 1994-2004, 2000.

49. Schrier DJ, Imre KM: The effects of adenosine agonists on human neutrophil function. J Immunol 137: 3284-9, 1986.

50. Satoh M, Kuroda Y, Yoshida H, et al.: Induction of lupus autoantibodies by adjvants. J Autoimmun 21(1): 1-9, 2003.

51. Rapoport MJ, Sharabi A, Aharoni D, et al.: Amelioration of SLElike manifestations in (NZBxNZW) Fl mice following treatment with a peptide based on the complementarity determining region 1 of an autoantibody is associated with a down-regulation of apoptosis and of the pro-apoptotic factor JNK Kinase. Clin Immunol 117(3): 262-70, 2005.

52. Bannwarth B: Drug-induced musculoskeletal disorders. Drug Saf 30(1): 27-46, 2007. 